\title{
Pharmacological modulation of b-adrenoceptors as a new cardioprotective strategy for therapy of myocardial dysfunction induced by ischemia and reperfusion ${ }^{1}$
}

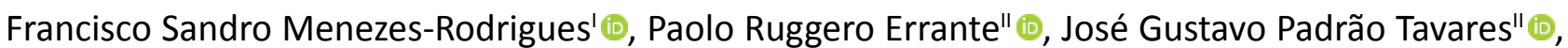

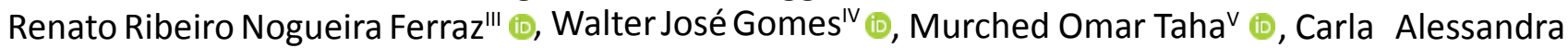
Scorza ${ }^{\mathrm{VI}}{ }_{(\mathbb{D}}$, Fúlvio Alexandre Scorza ${ }^{\mathrm{VI}}{ }^{(}$, Afonso Caricati-Neto $^{\mathrm{VII}}{ }_{(i)}$

'Fellow PhD degree, Postgraduate Program in Pharmacology, Department of Neurology and Neurosurgery, Universidade Federal de São Paulo (UNIFESP), Brazil. Conception and design of the study, analysis and interpretation of data, manuscript writing.

"Fellow PhD degree, Postgraduate Program in Pharmacology, UNIFESP, Sao Paulo-SP, Brazil. Manuscript writing.

'"'Fellow PhD degree, Postgraduate Program in Management of Health System (PMPA-GSS), Universidade Nove de Julho (UNINOVE), Sao Paulo-SP, Brazil. Statistics analysis.

IVPhD, Head, Division of Cardiovascular Surgery, UNIFESP, Sao Paulo-SP, Brazil. Technical procedures, interpretation of data, critical revision.

${ }^{\mathrm{P} P h D}$, Associate Professor, Department of Surgery, UNIFESP, Sao Paulo-SP, Brazil. Technical procedures, interpretation of data.

V'PhD, Associate Professor, Department of Neurology and Neurosurgery, UNIFESP, Sao Paulo-SP, Brazil. Analysis and interpretation of data, critical revision.

VIIPhD, Associate Professor, Department of Pharmacology, UNIFESP, Sao Paulo-SP, Brazil. Critical revision.

\section{Abstract}

Purpose: To evaluate the cardioprotective response of the pharmacological modulation of $\beta$-adrenergic receptors ( $\beta$-AR) in animal model of cardiac ischemia and reperfusion (CIR), in spontaneously hypertensive (SHR) and normotensive (NWR) rats.

Methods: CIR was induced by the occlusion of left anterior descendent coronary artery (10 $\mathrm{min}$ ) and reperfusion (75 $\mathrm{min}$ ). The SHR was treated with $\beta$-AR antagonist atenolol (AT, 10 $\mathrm{mg} / \mathrm{kg}$, IV) $5 \mathrm{~min}$ before CIR, and NWR were treated with $\beta$-AR agonist isoproterenol (ISO, 0.5 $\mathrm{mg} / \mathrm{kg}$, IV) 5 min before CIR.

Results: The treatment with AT increased the incidence of VA, AVB and LET in SHR, suggesting that spontaneous cardioprotection in hypertensive animals was abolished by blockade of $\beta$-AR. In contrast, the treatment with ISO significantly reduced the incidence of ventricular arrhythmia, atrioventricular blockade and lethality in NWR (30\%, 20\% and 20\%, respectively), suggesting that the activation of $\beta-A R$ stimulate cardioprotection in normotensive animals. Serum CK-MB were higher in SHR/CIR and NWR/CIR compared to respective SHAM group (not altered by treatment with AT or ISO).

Conclusion: The pharmacological modulation of $\beta-A R$ could be a new cardioprotective strategy for the therapy of myocardial dysfunctions induced by CIR related to cardiac surgery and cardiovascular diseases.

Key words: Cardiovascular Diseases. Myocardial Ischemia. Reperfusion. Receptors, Adrenergic. Drug Therapy. Rats. 


\section{Introduction}

Cardiovascular diseases (CVD) are the major cause of death in the world in developed and developing countries, and approximately 17 million people die each year from CVD and by 2030 this figure is expected to reach 26 million $^{1}$. Arterial hypertension constitutes an important risk factor for $C D^{2,3}$. Hypertension is characterized by systolic blood pressure equal to or above than $140 \mathrm{~mm} \mathrm{Hg}$ and/or diastolic blood pressure levels equal to or above than $90 \mathrm{mmHg}$.

Hypertension increases the risk of cardiac events, myocardium infarction and other ischemic cardiac diseases, which are treated by surgical interventions. These interventions involve the exposure of the heart for variable periods of cardiac ischemia and reperfusion (CIR). The increase of duration and severity of $\mathrm{CIR}$ are demonstrate to elicit increment of irreversible myocardial damage, and susceptibility to further injury during reperfusion, severely compromising cardiac structure and function ${ }^{4,5}$. Hypertension is characterized by autonomic dysfunctions, especially the sympathetic hyperactivity and involves tachycardia, increased peripheral vascular resistance, and elevated plasma catecholamine concentrations $^{6,7}$. Interestingly, some studies have suggested that sympathetic hyperactivity can be involved in cardioprotective response $\mathrm{e}^{8-10}$.

Following this line of reasoning, in vitro and in vivo studies using animal models of CIR showed that treatment with $\beta$-adrenoceptor $(\beta-A R)$ agonist isoproterenol (ISO) or ischemic preconditioning (IPC) protocol reduced infarct size, cardiac necrosis and ventricular arrhythmia (VA), reinforcing the concept of sympathetic mechanisms involvement in cardioprotection $^{8-11}$. In this sense, to investigate the role of $\beta$-AR in cardioprotection, we studied the effects of agonists and antagonist of $\beta-A R$ on the cardiac arrhythmias, lethality (LET) and serum levels of cardiac injury markers in spontaneously hypertensive (SHR) and normotensive (NWR) rats submitted to CIR.

\section{Methods}

All experimental protocols were approved by Ethical Committee of the UNIFESP (0065/12). The study enrolled adult male NWR and SHR weighting between 307 to $351 \mathrm{~g}$ with 12 to 14-week-old obtained from Centro de Desenvolvimento de Modelos Animais para Medicina e Biologia (CEDEME) of the Universidade Federal de São Paulo (UNIFESP). These animals were maintained under standard conditions of nutrition, hydration, temperature, light and humidity, and in accordance to normalization approved were.

The NWR and SHR were randomized into 8groups: 1) NWR/SHAM ( $n=12)$ : NWR submitted to surgical procedures, but not underwent CIR; 2) NWR/CIR ( $n=17)$ : NWR submitted to surgical procedure for CIR induction; 3) NWR/CIR+ISO $(n=12)$ : NWR submitted to surgical procedure for $\mathrm{CIR}$ induction and treated with $\beta$-AR agonist isoproterenol (ISO, $0.5 \mathrm{mg} / \mathrm{kg}$ administrated intravenously $5 \mathrm{~min}$ before of (IR); 4) NWR/ $\mathrm{CIR}+\mathrm{AT}+\mathrm{ISO}(\mathrm{n}=12)$ : NWR submitted to surgical procedure for $\mathrm{CIR}$ induction and treated with $\beta$-AR antagonist atenolol (AT, $10 \mathrm{mg} / \mathrm{kg}$ administrated intravenously $10 \mathrm{~min}$ before of $\mathrm{CIR})$ and ISO $(0.5 \mathrm{mg} / \mathrm{kg}$ administrated intravenously $5 \mathrm{~min}$ before of (IR); 5) SHR/ SHAM $(n=12)$ : SHR submitted to surgical procedures, but not underwent $\mathrm{CIR}$; 6) SHR/CIR ( $n=24):$ SHR submitted to surgical procedure for CIR induction; 7) SHR/CIR+AT $(n=12)$ : SHR submitted to surgical procedure for CIR induction and treated with AT $(10 \mathrm{mg} /$ $\mathrm{kg}$ administrated intravenously $5 \mathrm{~min}$ before of $\mathrm{CIR}$; ; 8) SHR/CIR+ISO (n=6): SHR submitted to surgical procedure for $\mathrm{CIR}$ induction and treated with ISO $(0.5 \mathrm{mg} / \mathrm{kg}$ administrated intravenously $5 \mathrm{~min}$ before $\mathrm{CIR}$ ).

\section{Non-invasive method of measuring blood pressure}

Systolic blood pressure (SBP) in nonanesthetized both NWR and SHR was measured 
by non-invasive tail-cuff plethysmography using the "Mouse and Rat Tail Cuff Method Blood Pressure System" (System IITC Inc., California, USA), in accordance with method described by Musial et al ${ }^{12}$.

\section{Method for induction of $C I R$}

The method for induction of $\mathrm{CIR}$ has previously been described by our lab ${ }^{11,13-}$ 15. Rats were anesthetized with urethane $(1.25 \mathrm{~g} / \mathrm{kg})$, and fixed in the supine position. After intubation (Jelco 14G, USA), rats were mechanically ventilated using a mechanic ventilator Insight model EFF 312 (Insight Equipamentos Científicos, Ribeirao Preto$\mathrm{SP}$, Brazil). After stabilization for $15 \mathrm{~min}$, thoracotomy was performed to place the vascular tourniquet (4/0 braided silk suture attached to a $10-\mathrm{mm}$ micropoint reversecutting needle, Ethicon $\mathrm{K}-890 \mathrm{H}$, USA) around the left anterior descending coronary artery to induce ischemia. After of $10 \mathrm{~min}$ of cardiac ischemia, the tourniquet was removed to allow coronary recirculation for $75 \mathrm{~min}$ (cardiac reperfusion). The cardiac electrical activity in all groups studied was monitored by electrocardiogram (ECG) system using a method previously described by our lab ${ }^{11,13-15}$. ECG analysis was performed during $100 \mathrm{~min}$ of duration (stabilization for $15 \mathrm{~min}$, cardiac ischemia for $10 \mathrm{~min}$ and cardiac reperfusion for $75 \mathrm{~min}$ ). The ECG was recorded using a biopotential amplifier by means of needle electrodes placed subcutaneously on the limbs. Successful surgical obstruction of the coronary artery was validated by ECG alterations (increase in R wave and ST segment) caused by cardiac ischemia. The body temperature was maintained at $37.5 \circ \mathrm{C}$ with a heated operating platform and appropriate heating lamps and was evaluated routinely via a rectal thermometer.

Evaluation of cardiac function by ECG analysis

The ECG data were recorded using an acquisition system AqDados 7.02 (Lynx Tecnologia Ltda., Brazil), an acquisition system AqDados 7.02 (Lynx Tecnologia Ltda., Brazil), and analyzed using the software AqDAnalysis 7 (Lynx Tecnologia Ltda., Brazil). Using this software, the heart rates were evaluated, as well as incidence of ventricular arrhythmias (VA), atrioventricular block (AVB) and lethality (LET), in response to CIR. The ventricular fibrillation, torsades de pointes, and ventricular tachycardia parameters were considered only as VA.

\section{Evaluation of cardiac lesion biomarkers}

After ECG recording, blood sample were collected from the abdominal aorta artery, and centrifuged $\left(2.500 \mathrm{rpm}\right.$, for $40 \mathrm{~min}$, at $5^{\circ} \mathrm{C}$ ) for serum isolation. The serum was stored at $-20^{\circ} \mathrm{C}$ for biochemical analysis. Quantitative determination of creatine kinase $M B$ fraction (CK-MB) was performed using a kineticUV method, measured at $340 \mathrm{~nm}$ (Kit Katal Biotecnológica Ind. Com. Ltda., Belo HorizonteMG, Brazil).

\section{Statistical analysis}

The incidence of VA, AVB, and LET were statistically evaluated using the Fisher's exact test $(p<0.05)$. Ratio between heart mass and body mass (HM/BM) was statistically evaluated using Student's test ( $p<0.05)$, and serum concentration of CK-MB are expressed as mean \pm standard error of mean (SEM), and analysis of variance (ANOVA) was applied, followed by the Tukey post-test $(p<0.05)$ using Prism 5.0 software (GraphPad, USA).

\section{Results}

Blood pressure and cardiac mass in SHR and NWR

The systolic blood pressure (SBP) value was $199 \pm 4 \mathrm{~mm} \mathrm{Hg}$ in SHR and 132 $\pm 2 \mathrm{~mm} \mathrm{Hg}$ in NWR. Cardiac hypertrophy 
represents another phenotypic characteristic of hypertension. In this work, the cardiac mass was $1,335 \pm 110 \mathrm{mg}$ in SHR and 1,121 \pm 98 $\mathrm{mg}$ in NWR, showing that cardiac mass in SHR was $19 \%$ greater compared to NWR (figure 1 ). Although the body mass value was $11 \%$ lower
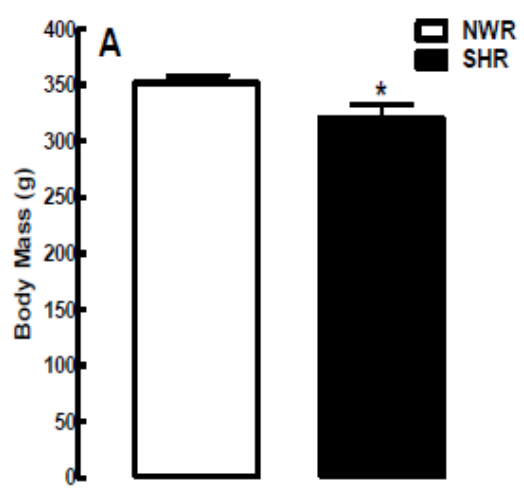

Figure 1 - Histogram showing the values of body mass (A) and heart mass ratio and body mass (HM/BM) (B) in NWR and SHR. The results are expressed as mean \pm standard error of the mean, and Student's test was applied to evaluate the statistically different from the NWR, ${ }^{*} p<0.05$ ( $n=40$ samples for each group).

\section{Cardiac function in SHR and NWR}

ECG analysis showed that the incidence of VA, AVB and LET was $0 \%$ in SHR/SHAM and NWR/SHAM groups (Fig. 2). The incidence of VA, AVB and LET in SHR/CIR group was $40 \%$, $20 \%$ and $20 \%$, respectively, and in NWR/CIR group (85\%, 80\% and $70 \%$, respectively (Fig. 2 ). These results show that the incidence of VA, AVB and LET significantly lower in SHR compared to NWR, suggesting the presence of spontaneous adaptive cardioprotective response in hypertensive. To test this hypothesis, we studied the effects of drugs that interfere on the activity of cardiac $\beta-A R$ in SHR and NWR. It is well known that hypertension in SHR is related to sympathetic hyperactivity ${ }^{16-18}$. Then, we treated the SHR with AT before CIR to reduce this sympathetic hyperactivity due to blockade of the $\beta-A R$ in cardiovascular tissues. We observed that the AT significantly increased the incidence of VA (from $40 \%$ to $80 \%$ ), AVB (from $20 \%$ to $83 \%$ ), and LET (from $20 \%$ to $71 \%$ ) in SHR/CIR group (Fig. 2). These results indicate that pharmacological blockade in SHR (320 $\pm 13 \mathrm{~g}$ ) compared to NWR (351 \pm $7 \mathrm{~g})$, the ratio between heart mass and body mass (HM/BM) was 31\% higher in SHR (0.0041) compared to NWR (0.0031). These phenotypic characteristics confirm the hypertensive state in SHR (Fig. 1).
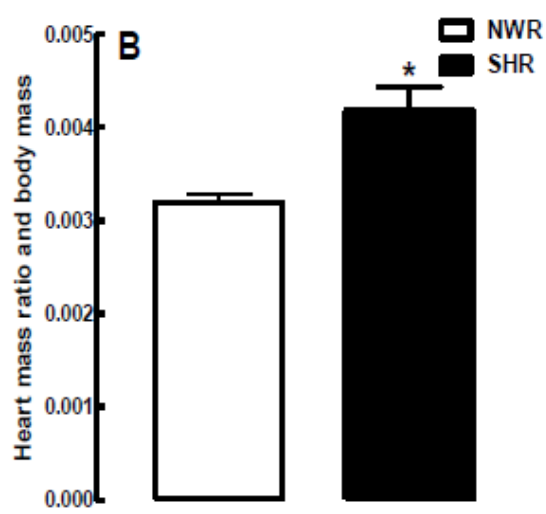

of $\beta$-AR abolished the spontaneous adaptive cardioprotective response in hypertensive animals, indicating the direct involvement of the $\beta-A R$ in this response.

To confirm the involvement of the $\beta-A R$ in sympathetic mechanisms involved in the cardioprotective response, we treated the NWR with ISO before CIR to increase the sympathetic activity, simulating the sympathetic hyperactivity in SHR. We observed that this treatment significantly reduced the incidence of VA (from $85 \%$ to $30 \%$ ), AVB (from $80 \%$ to $30 \%$ ), and LET (from $70 \%$ to $30 \%$ ) in NWR/CIR group (Fig. 2), indicating that the stimulation of the cardiac $\beta$-AR in normotensive animals induces cardioprotective response. To confirm this hypothesis, the NWR/CIR group was treated with AT before of the stimulation of $\beta$-AR by ISO. These assays showed that the pretreatment with $A T$ abolished the reduction in the incidence of VA, AVB and LET induced by ISO in NWR/CIR group (Fig. 2), indicating that the blockade of cardiac $\beta$-AR abolished the cardioprotective response induced by pharmacological stimulation of these receptors 
in normotensive animals. The results obtained in NWR and SHR submitted to CIR confirm the involvement of the cardiac $\beta-A R$ in the cardioprotective response (Fig. 2).
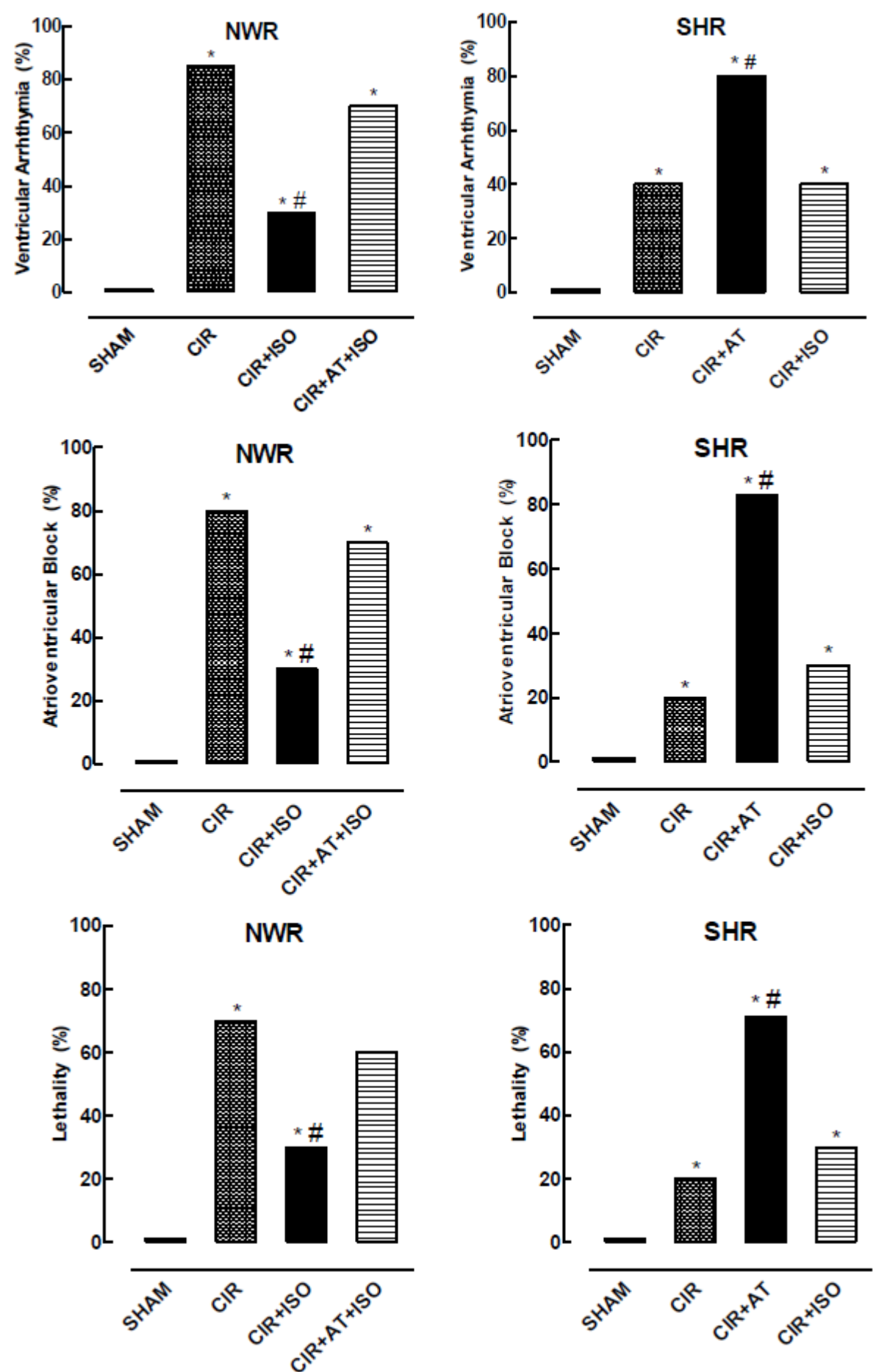

Figure 2 - Incidence of ventricular arrhythmias (AV), atrioventricular block, and lethality in the NWR/SHAM, NWR/CIR, NWR/CIR+ISO, NWR/CIR+AT+ISO, SHR/SHAM, SHR/CIR, SHR/CIR+AT, NWR/CIR+ISO groups. The results are expressed as mean, and Fisher's exact test was applied. * Statistically different from the NWR/ SHAM or NWR/CIR and SHR/SHAM or SHR/CIR, $p<0.05$. \# Statistically different from the $\mathrm{CIR}, \mathrm{p}<0.05 .(\mathrm{n}=12$ to 24 for each group). 


\section{Cardiac lesion biomarker in SHR and NWR}

The biochemical evaluation of cardiac injury biomarker CK-MB showed important differences between SHR and NWR in response to $\mathrm{CIR}$ and the treatment with $A T$ or ISO. Serum concentration of CK-MB were significantly higher in the NWR/CIR compared to NWR/SHAM group (Fig. 3), indicating that $\mathrm{CIR}$ produced an increase in the serum concentration of $\mathrm{CK}-\mathrm{MB}$ in normotensive animals. In contrast, serum concentration of CK-MB was similar in the SHR/SHAM and SHR/ $\mathrm{CIR}$ (Fig. 3), indicating that CIR was not able to increase the serum concentration of CK$\mathrm{MB}$ in hypertensive animals. Comparisons of serum concentration of CK-MB between SHR/ SHAM and NWR/SHAM groups, showed that CK-MB is increased in hypertensive animals independently of CIR (Fig. 3), suggesting that hypertension could stimulate the increase in the serum concentration of CK-MB. However, the involvement of sympathetic mechanisms in this response is unclear.

To test the possible involvement of sympathetic mechanisms in the serum levels of CK-MB, we studied the effects of AT and ISO on the serum concentration of this cardiac injury biomarker in NWR and SHR submitted to CIR. We observed that the pretreatment with AT not altered serum concentration of CK-MB in SHR/CIR group (Fig. 3), indicating that the $\beta-A R$ does not interfered in the synthesis of CK-MB in hypertensive animals. We also studied the effects of the treatment with ISO on the serum levels of CK-MB in NWR submitted to CIR. This treatment does not alter serum levels of CK$\mathrm{MB}$ compared to NWR/CIR (Fig. 3), indicating that the $\beta-A R$ does not interfered in the serum concentration of $\mathrm{CK}-\mathrm{MB}$ in normotensive animals.
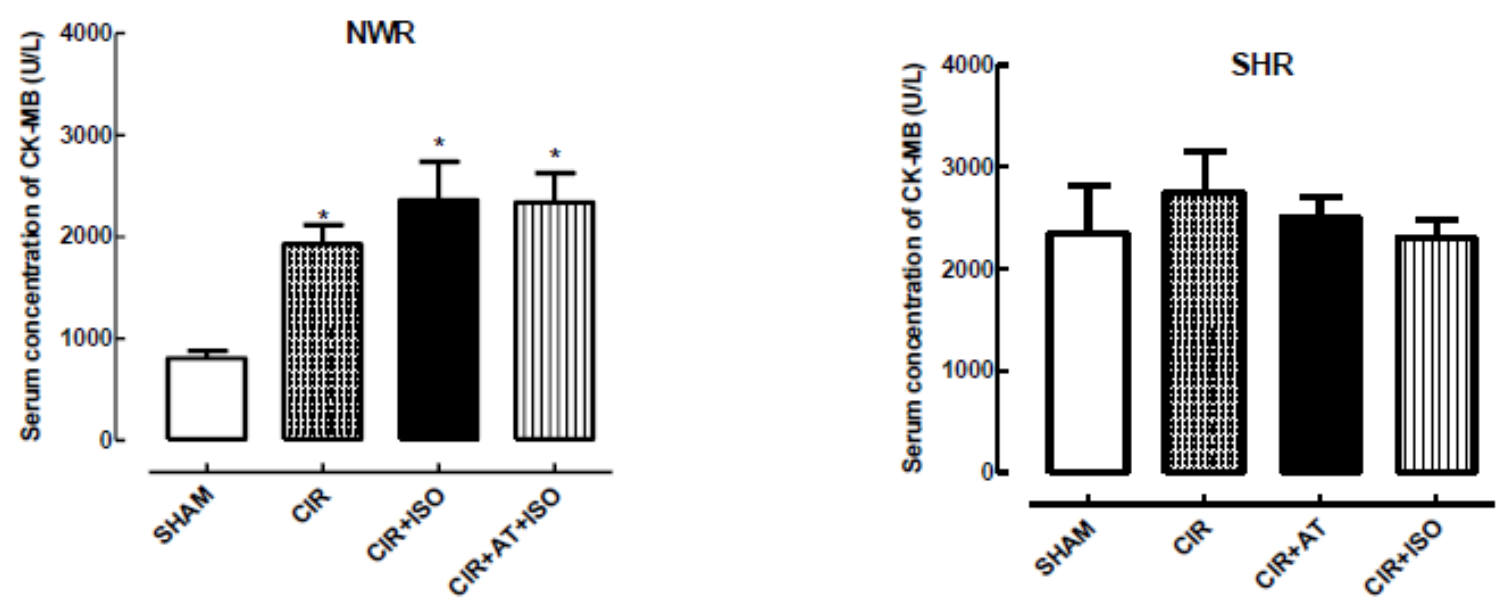

Figure 3 - Serum concentration of CK-MB in the NWR/SHAM, NWR/CIR, NWR/CIR+ISO, NWR/CIR+AT+ISO, SHR/SHAM, SHR/CIR, SHR/CIR+AT, NWR/CIR+ISO groups. The results are expressed as mean \pm standard error of the mean, and analysis of variance (ANOVA) was applied, followed by the Tukey post-test. * Statistically different from the NWR/SHAM or NWR/CIR and SHR/SHAM or SHR/CIR, $p<0.05$. "Statistically different from the $\mathrm{CIR}, \mathrm{p}<0.05$. ( $n=6$ to 8 samples for each group).

\section{Discussion}

Using in vivo animal model of $\mathrm{CIR}$, we investigated in the present study the involvement of cardiac $\beta-A R$ in the cardioprotective response in normotensive (NWR) and hypertensive (SHR) animals. This study showed that the CIR increases the 
incidence of VA, AVB and LET in NWR and SHR, but the incidences of all these parameters were significantly lower in SHR. These findings suggest, for the first time, the presence of a spontaneous adaptive cardioprotective response in hypertensive animals. The SHR strain is the main study model of primary hypertension in humans ${ }^{19}$. In this model, the hypertension is related to changes in cardiovascular parameters, determinants of systemic arterial pressure, with increased total peripheral resistance with normal or decreased cardiac output ${ }^{20}$. The elevation of blood pressure levels in SHR begins approximately in the 5th or 6th week of life, presenting high pressure levels between the 7th to the 15th weeks and plateau around the 20th to the 28th week, at which age, the blood pressure values reach their maximum levels. Due to the onset of hypertension, the SHR model develops progressive cardiac hypertrophy that progresses to heart failure ${ }^{21,22}$. Similarly to human hypertension, the SHR used in present work presented SBP values above $140 \mathrm{~mm}$ $\mathrm{Hg}$ and cardiac hypertrophy, confirming the hypertensive state in $\mathrm{SHR}^{19}$.

Because of the hypertension, the SHR develops cardiac hypertrophy that progresses to heart failure ${ }^{21,22}$. However, the main triggering factor of hypertensive conditions in the SHR is the increase in peripheral vascular resistance due to sympathetic hyperactivity, one of the main features of these animals ${ }^{23}$. Therefore, SHR presents a significant reduction in microvascular density and blood supply in several organs, like skeletal muscle ${ }^{24}$, gut ${ }^{25}$, skin $^{26}$ and heart ${ }^{27}$. The reduced density of myocardial capillaries leads to the inability of microcirculatory growth, which does not accompany the cardiac hypertrophy triggered by the hypertension ${ }^{28}$.

It is well established that the hyperactivity sympathetic is involved in the pathogenesis of hypertension in humans and animal hypertension models, such as $\mathrm{SHR}^{16-}$ 18. For example, the sympathetic activity and discharge from cervical, splanchnic and renal sympathetic neurons is significantly increased in $\mathrm{SHR}^{16-18}$. This sympathetic hyperactivity is also involved in other cardiovascular dysfunctions, including the acute myocardial infarction (AMI). However, a growing number of evidences suggest that the sympathetic hyperactivity associated to cardiovascular diseases could stimulate the cardioprotective response ${ }^{8-11,29}$. However, the molecular mechanisms involved in this cardioprotective response remains unclear.

To investigate the molecular mechanisms involved in this cardioprotective response, we studied the effects of agonist (ISO) and antagonist (AT) of $\beta$-AR on the incidence of VA, AVB and LET in SHR submitted to $\mathrm{CIR}$. The treatment with $\beta_{1}-A R$ antagonist AT increased the incidence of VA, AVB and LET in SHR submitted to CIR, confirming that the blockade of cardiac $\beta_{1}-A R$ abolished the spontaneous cardioprotection in hypertensive animals. The treatment with $\beta_{1} / \beta_{2}-A R$ agonist ISO does not change the incidence of VA, AVB and LET in SHR submitted to CIR, probably due to preexistent spontaneous cardioprotective response in hypertensive animals. These results suggest that sympathetic hyperactivity associated to primary hypertension could induce a set of adaptive responses able to attenuate myocardial dysfunctions and lesions caused by CIR, including an increased density/ function of cardiac $\beta-A R$.

It is important to mention that prior exposure to brief $\mathrm{CIR}$ periods conferred spontaneous cardioprotection against cardiac lesions produced by prolonged $\mathrm{CIR}^{8}$. This procedure called ischemic preconditioning (IPC) has become one of the experimental nonpharmacological strategies to reduce the area of injury produced by CIR. In addition, it has been shown that IPC can confer cardioprotection by 
decreasing the incidence of arrhythmias caused by $\mathrm{CIR}^{11,30,31}$. These events are characteristic of IPC that is able to provide cardioprotection, the cardioprotective effects cause by was observed in several types of cardiac preparations (culture of cardiomyocytes and isolated hearts) and animal species, among which are the rats ${ }^{11,32-}$ ${ }^{34}$. Data from the literature have shown that blocking $\beta_{1}$-AR decreases cardioprotection promoted by IPC, one of which such blocking promotes negative effects on cardiac tolerance to IPC, since this blockade can exert negative effects on functional CIR tolerance and signaling pathways involved in the cardioprotection caused by activation of $\beta_{1}-A R$ and IPC ${ }^{35-39}$.

In vitro studies demonstrated that prior exposure for 1 to 2 short cycles of ischemia ( $5 \mathrm{~min}$ ) and reperfusion (10 $\mathrm{min}$ ) reduced the incidence of ventricular fibrillation from $42 \%$ to $17 \%$ with 2 cycles and completely abolished this type of arrhythmia with 1 cycle in isolated rabbit hearts submitted to ischemia for 30 min followed by reperfusion for $45 \mathrm{~min}^{32}$. In addition, it was showed that the pre-exposure of hearts isolated from Wistar rats to 1 cycle of ischemia (5 $\mathrm{min}$ ) and reperfusion (5 $\mathrm{min}$ ) reduced the number of premature ventricular beats (518 $\pm 71 \mathrm{bpm}$ for $195 \pm 40 \mathrm{bpm}$ ) and reduced the incidence (from $100 \%$ to $25 \%$ ) and duration (from $44 \pm 9$ s to $18 \pm 5 \mathrm{~s}$ ) of ventricular tachycardia in isolated hearts submitted to 30 min of ischemia ${ }^{40}$. The cardioprotective efficacy of IPC by prior exposure to 2 cycles of ischemia (5 min) and reperfusion (5 min) in Wistar rats submitted to cardiac ischemia for $30 \mathrm{~min}$ and reperfusion for $24 \mathrm{~h}$ significantly reduced incidence of AV and LET (from 50\% to $12 \%$ ), as well as the area of the myocardial injury (from $40 \%$ to $25 \%)^{34}$.

To confirm the involvement of $\beta$-AR in cardioprotective response, we also studied the effects of ISO and AT on the incidence of VA, AVB and LET in NWR submitted to CIR. The treatment with ISO significantly reduced the incidence of VA, AVB and LET in NWR submitted to CIR, suggesting that the pharmacological activation of $\beta$-AR stimulated the cardioprotective response in normotensive animals. This effect of ISO was abolished by the pretreatment with AT, confirming that the blockade of cardiac $\beta-A R$ abolished the pharmacological cardioprotective response in NWR.

Several experimental studies have suggested the involvement of different subtypes of $\beta$-AR in the cardioprotective response $e^{29,41}$. It was showed that the treatment with the $\beta_{2}$ AR agonist clenbuterol produced a significant cardioprotective response against CIR injury by the $\beta_{2}-A R-G i-p r o t e i n$ signaling stimulation in rats ${ }^{29}$. The transient activation of $\beta_{1} / \beta_{2}-A R$ in hearts preconditioned for 5 min with agonists of $\beta_{1} / \beta_{2}-A R$ (ISO), $\beta_{1}$-AR (denopamine), $\beta_{2}-$ $A R$ (formoterol) elicited cardioprotective response against CIR injury in rats ${ }^{41}$. This study also suggested the involvement of nitric oxide (NO) in $\beta_{1} / \beta_{2}-A R$ ischemic preconditioning (IPC) and the Gi protein in $\beta_{2}-A R$ mediated preconditioning in rats ${ }^{41}$. Salie et al. ${ }^{41}$ suggested that the intracellular messengers coupled to $\beta-A R$ such as protein kinase A (PKA) and PI3-K is essential for cardioprotective response mediated by cardiac $\beta_{1} / \beta_{2}-A R$.

The selective stimulation of $\beta_{3}-A R$ is able to reduce infarct size in animal model of CIR. The pretreatment with the $\beta_{3}-A R$ agonist BRL37344 $(5 \mu \mathrm{g} / \mathrm{kg}$ ) reduce infarct size in wild-type mice with left ventricular function improved in $\beta_{3}$ $A R$ agonist treated mice. The incubation with $\beta_{3}-A R$ agonist significantly reduces cell death in isolated adult mouse cardiomyocytes during hypoxia-reoxygenation periods and decreased susceptibility to deleterious opening of the mitochondrial permeability transition pore, via a mechanism dependent on the Akt-NO signaling pathway. The administration of BRL37344 decrease infarct size and improved long-term left ventricular contractile function ${ }^{42}$. 
In addition, we observed that the serum concentration of CK-MB was significantly higher in the NWR submitted to CIR compared to its respective SHAM, suggesting that CIR induced cardiac injury. In contrast, serum concentration of CK-MB was similar in the SHR submitted to CIR and SHR/SHAM. Comparisons of serum concentration of CK-MB between SHR submitted to CIR and NWR/SHAM showed that the serum concentration of CK-MB is increased in hypertensive independently of CIR, suggesting that hypertension could stimulate the increase of CK-MB. These results agree with the results showed in the literature in high performance athletes, which according to our thesis these athletes present the IPC-induced cardioprotection similarly to those observed in hypertensives ${ }^{43-45}$. To test the possible involvement of sympathetic mechanisms in the serum levels of cardiac injury biomarker, we studied the effects of AT or ISO on the serum concentration of CK-MB. To reduce sympathetic hyperactivity in SHR, the cardiac $\beta$-AR was blocked by AT administration before $\mathrm{CIR}$. In the SHR, the treatment with AT before CIR does not alter serum levels of CK-MB. These results suggest that blockade of $\beta-A R$ does not interfere in the synthesis of cardiac injury markers in hypertensive animals. The treatment with ISO to increase the sympathetic activity does not alter serum levels of CK-MB in NWR submitted to CIR. Thus, these findings could lead to therapeutic strategies to prevent both cardiac arrhythmias and mortality in AMI in hypertensive patients. In addition, this strategy could be used in cardiac surgery to prevent the myocardial dysfunction induced by CIR.

\section{Conclusion}

These results support the concept that $\beta-A R$ are involved in cardioprotective response. Therefore, pharmacological modulation of these receptors could be a new cardioprotective strategy for the therapy of myocardial dysfunction induced by CIR related to cardiac surgery and cardiovascular diseases in humans, such as ischemic cardiac diseases and primary hypertension.

\section{References}

1. WHO. World Health Organization. 2013. Available from: http://www.who.int

2. Kaplan RC. Treatment of hypertension to prevent stroke: translating evidence into clinical practice. J Clin Hypertens. 2001;3(3):153-64. PMID: 11416700.

3. Anwer Z, Sharma RK, Garg VK, Kumar N, Kumari A. Hypertension management in diabetic patients. Eur Rev Med Pharmacol Sci. 2011;15(11):1256-63. doi: 10.1212/01. wnl.0000176058.09848.bb.

4. Verma S, Fedak PW, Weisel RD, Butany J, Rao V, Maitland A, Li RK, Dhillon B, Yau TM. Fundamentals of reperfusion injury for the clinical cardiologist. Circulation. 2002;105(20):2332-6. PMID: 12021216.

5. Binder A, Ali A, Chawla R, Aziz HA, Abbate A, Jovin IS. Myocardial protection from ischemia-reperfusion injury post coronary revascularization. Expert Rev Cardiovasc Ther. 2015;13(9):1045-57. PMID: 26202544.

6. Schömig A, Dart AM, Dietz R, Mayer E, Kübler $W$. Release of endogenous catecholamines in the ischemic myocardium of the rat. Part A: locally mediated release. Circ Res. 1984;55(5):689-701. PMID: 6488489.

7. Kitagawa $\mathrm{H}$, Yamazaki T, Akiyama T, Yahagi $\mathrm{N}$, Kawada T, Mori H, Sunagawa K. Modulatory effects of ketamine on catecholamine efflux from in vivo cardiac sympathetic nerve endings in cats. Neurosci Lett. 2002;324(3):232-6. PMID: 12009530.

8. Murry CE, Jennings RB, Reimer KA. Preconditioning with ischemia: a delay of lethal cell injury in ischemic myocardium. Circulation. 1986;74(5):1124-36. PMID: 3769170.

9. Bankwala Z, Hale SL, Kloner RA. Alpha-adrenoceptor stimulation with exogenous norepinephrine or release of endogenous catecholamines mimics ischemic preconditioning. Circulation. 
1994;90(2):1023-8. PMID: 8044915.

10.Wever KE, Hooijmans CR, Riksen NP, Sterenborg TB, Sena ES, RitskesHoitinga $M$, Warlé MC. Determinants of the efficacy of cardiac ischemic preconditioning: a systematic review and meta-analysis of animal studies. PloS One. 2015;10(11):e0142021. doi: 10.1371/ journal.pone.0142021.

11. Tavares JGP, Errante PR, Govato TCP, Vasques ÊR, Ferraz RRN, Taha MO, Menezes-Rodrigues FS, Caricati-Neto A Cardioprotective effect of preconditioning is more efficient than postconditioning in rats submitted to cardiac ischemia and reperfusion. Acta Cir Bras. 2018;33(7):588-96. doi: 10.1590/ s0102-865020180070000004.

12.Musial DC, Bomfim GH, Miranda-Ferreira R, Caricati-Neto A, Jurkiewicz A, Jurkiewicz NH. Chronic treatment with red wine modulates the purinergic neurotransmission and decreases blood pressure in hypertensive SHR and diabetic-STZ rats. Int J Food Sci Nutr. 2015;66(5):579-86. doi: 10.3109/09637486.2015.1056110.

13.Tavares JGP, Vasques ER, Arida RM, Cavalheiro EA, Cabral FR, Torres LB, MenezesRodrigues FS, Jurkiewicz A, Caricati-Neto A, Godoy CM, Gomes da Silva S. Epilepsyinduced electrocardiographic alterations following cardiac ischemia and reperfusion in rats. Braz J Med Biol Res. 2015;48(2):1405. doi: 10.1590/1414-431X20144311.

14.Tavares JGP, Menezes-Rodrigues FS, Vasques ER, Reis MCM, Paula L. A Simple and efficient methodology for the study of cardioprotective drugs in animal model of cardiac ischemia-reperfusion. J Mol Imag Dyn. 2017;7(133):2. doi: 10.4172/21559937.1000133.

15. Menezes-Rodrigues FS, Errante PR, Ferreira RM, Tavares JGP, Paula L de, Araújo EA, Govato TCP, Tikazawa EH, Reis MDCM, Luna-Filho B, Ferraz RRN, Oliveira-Júnior IS, Taha MO, Caricati-Neto A. Cardioprotective effect of lipstatin derivative orlistat on normotensive rats submitted to cardiac ischemia and reperfusion. Acta Cir Bras. 2018;33(6):524-32. doi: 10.1590/s0102865020180060000007.

16.Okamoto K, Nosaka S, Yamori Y, Matsumoto M. Participation of neural factor in the pathogenesis of hypertension in the spontaneously hypertensive rat. Jpn Heart J. 1967;8(2):168-80. PMID: 5299130.

17.Judy WV, Watanabe AM, Murphy WR, Aprison BS, Yu PL. Sympathetic nerve activity and blood pressure in normotensive backcross rats genetically related to the spontaneously hypertensive rat. Hypertension. 1979;1(6):598-604. PMID: 541052.

18.Judy WV, Farrell SK. Arterial baroreceptor reflex control of sympathetic nerve activity in the spontaneously hypertensive rat. Hypertension. 1979;1(6):605-14. PMID: 541053.

19.Gheorghe A, Griffiths U, Murphy A, LegidoQuigley H, Lamptey P, Perel P. The economic burden of cardiovascular disease and hypertension in low-and middle-income countries: a systematic review. BMC Public Health. 2018;18(1):975. doi: 10.1186/ s12889-018-5806-x.

20.Zhou X, Frohlich ED. Analogy of cardiac and renal complications in essential hypertension and aged SHR or L-NAME/SHR. Med Chem. 2007;3(1):61-5. PMID: 17266625.

21.Bing $\mathrm{OH}$, Conrad $\mathrm{CH}$, Boluyt $\mathrm{MO}$, Robinson KG, Brooks WW. Studies of prevention, treatment and mechanisms of heart failure in the aging spontaneously hypertensive rat. Heart Fail Rev. 2002;7(1):71-88. PMID: 11790924.

22.Pravenec $M$, Křen $V$, Landa $V$, Mlejnek $P$, Musilová A, Šilhavỳ J, Šimáková M, Zídek $\mathrm{V}$. Recent progress in the genetics of spontaneously hypertensive rats. Physiol Res. 2014;63 Suppl 1:s1-8. PMID: 24564651.

23. Fazan VPS, Salgado HC, Barreira AA. Aortic depressor nerve unmyelinated fibers in spontaneously hypertensive rats. Am J Physiol-Heart Circ Physiol. 2001;280(4):H1560-4. doi: 10.1152/ ajpheart.2001.280.4.H1560.

24.le Noble FA, Stassen FR, Hacking WJ, Boudier HAS. Angiogenesis and hypertension. J Hypertens. 1998;16(11):1563-72. PMID: 9856355.

25. Henrich $H$, Hertel $R$, Assmann $R$. Structural differences in the mesentery microcirculation between normotensive and spontaneously hypertensive rats. Pflüg Arch. 1978;375(2):153-9. PMID: 567786.

26. Haack DW, Schaffer JJ, Simpson JG. Comparisons of cutaneous microvessels 
from spontaneously hypertensive, normotensive Wistar-Kyoto, and normal Wistar rats. Proc Soc Exp Biol Med. 1980;164(4):453-8. PMID: 7413666.

27.Sabri A, Samuel J-L, Marotte F, Poitevin P, Rappaport L, Levy BI. Microvasculature in angiotensin II-dependent cardiac hypertrophy in the rat. Hypertension. 1998;32(2):371-5. PMID: 9719070.

28.Feihl F, Liaudet L, Waeber B, Levy BI. Hypertension: a disease of the microcirculation? Hypertension. 2006;48(6):1012-7. doi: 10.1161/01. HYP.0000249510.20326.72.

29.Zhang Q, Xiang J, Wang X, Liu H, Hu B, Feng $\mathrm{M}, \mathrm{Fu} \mathrm{Q}$. Beta(2)-adrenoceptor agonist clenbuterol reduces infarct size and myocardial apoptosis after myocardial ischaemia/reperfusion in anaesthetized rats. Br J Pharmacol. 2010;160(6):1561-72. doi: 10.1111/j.1476-5381.2010.00813.x.

30.Li J, Zheng $H$, Du $H$, Tian $X$, Jiang $Y$, Zhang $S$, Kang Y, Li X, Chen J, Lu C, Lai ZH, Liang FR. The multiple lifestyle modification for patients with prehypertension and hypertension patients: a systematic review protocol. BMJ Open. 2014;4(8):e004920. doi: 10.1136/ bmjopen-2014-004920.

31.Rubino A, Yellon DM. Ischaemic preconditioning of the vasculature: an overlooked phenomenon for protecting the heart? Trends Pharmacol Sci. 2000;21(6):225-30. PMID: 10838610.

32.Lukas A, Botsford MW. Cardioprotection induced by ischemic preconditioning in the mammalian heart: effects on arrhythmogenesis. Can J Physiol Pharmacol. 1997;75(4):316-25. PMID: 9196858.

33. Balakumar P, Rohilla A, Singh G, Singh K, Singh M. Modulation of cardioprotective effect of ischemic pre-and postconditioning in the hyperhomocysteinemic rat heart. Methods Find Exp Clin Pharmacol. 2009;31(2):71-9. doi: 10.1358/mf.2009.31.2.1357292.

34.Kolettis TM, Vilaeti AD, Tsalikakis DG, Zoga A, Valenti M, Tzallas AT, Papalois A, Iliodromitis EK. Effects of pre-and postconditioning on arrhythmogenesis in the in vivo rat model. J Cardiovasc Pharmacol Ther. 2013;18(4):37685. doi: $10.1177 / 1074248413482183$.

35.Nasa Y, Yabe K, Takeo S. $\beta$-Adrenoceptor stimulation-mediated preconditioning-like cardioprotection in perfused rat hearts. J
Cardiovasc Pharmacol. 1997;29(4):436-43. PMID: 9156351.

36. Frances C, Nazeyrollas P, Prevost A, Moreau F, Pisani J, Davani S, Kantelip JP, Millart H. Role of beta 1-and beta 2-adrenoceptor subtypes in preconditioning against myocardial dysfunction after ischemia and reperfusion. J Cardiovasc Pharmacol. 2003;41(3):396-405. PMID: 12605018.

37.Spear JF, Prabu SK, Galati D, Raza H, Anandatheerthavarada HK, Avadhani NG. Beta 1-adrenoreceptor activation contributes to ischemia-reperfusion damage as well as playing a role in ischemic preconditioning. Am J Physiol-Heart Circ Physiol. 2007;292(5):H2459-66. doi: 10.1152/ajpheart.00459.2006.

38. Maier T, Schreckenberg R, Schlüter K-D. Effect of preischemic $\beta$-adrenoceptor stimulation on postischemic contractile dysfunction. Life Sci. 2009;84(13-14):43743. doi: 10.1016/j.Ifs.2009.01.006.

39. Hoe LES, Schilling JM, Busija AR, Haushalter KJ, Ozberk V, Keshwani MM, Roth DM, ToitED, Headrick JP, Patel HH, Peart JN. Chronic $\beta 1$ adrenoceptor blockade impairs ischaemic tolerance and preconditioning in murine myocardium. Eur J Pharmacol. 2016;789:17. doi: 10.1016/j.Ifs.2009.01.006.

40.Matejíková J, Kucharská J, Pintérová M, Pancza D, Ravingerová T. Protection against ischemia-induced ventricular arrhythmias and myocardial dysfunction conferred by preconditioning in the rat heart: involvement of mitochondrial KATP channels and reactive oxygen species. Physiol Res. 2009;58(1). PMID: 18198991.

41.Salie $R$, Moolman JA, Lochner A. The role of $\beta$-adrenergic receptors in the cardioprotective effects of betapreconditioning $(\beta P C)$. Cardiovasc Drugs Ther. 2011;25(1):31-46. doi: 10.1007/ s10557-010-6275-3.

42.García-Prieto J, García-Ruiz JM, SanzRosa D, Pun A, García-Alvarez A, Davidson $S M$, Fernández-Friera $L$, Nuno-Ayala $M$, Fernández-Jiménez R, Bernal JA, IzquierdoGarcia JL, Jimenez-Borreguero J, Pizarro G, Ruiz-Cabello J, Macaya C, Fuster V, Yellon DM, Ibanez B. $\beta 3$ adrenergic receptor selective stimulation during ischemia/ reperfusion improves cardiac function in translational models through inhibition of 
MPTP opening in cardiomyocytes. Basic Res Cardiol. 2014;109(4):422. doi: 10.1007/ s00395-014-0422-0.

43. Noakes TD, Higginson L, Opie LH. Physical training increases ventricular fibrillation thresholds of isolated rat hearts during normoxia, hypoxia and regional ischemia. Circulation. 1983;67(1):24-30. PMID: 6291806.

44.Weippert M, Behrens K, Rieger A, Kumar
M, Behrens M. Effects of breathing patterns and light exercise on linear and nonlinear heart rate variability. Appl Physiol Nutr Metab. 2015;40(8):762-8. doi: 10.1139/ apnm-2014-0493.

45. Emokpae MA, Nwagbara GO. Serum creatine kinase-MB isoenzyme activity among subjects with uncomplicated essential hypertension: any sex differences. Med Sci. 2017;5(2):8. doi: 10.3390/medsci5020008.

\section{Correspondence:}

Afonso Caricati Neto

Laboratório de Farmacologia Autonômica e Cardiovascular, UNIFESP

Rua Pedro de Toledo, 699/70 andar

04039-032 São Paulo - SP Brasil

caricatineto@gmail.com

Received: Jan 22, 2019

Review: Mar 26, 2019

Accepted: Apr 25, 2019

\section{Conflict of interest: none}

Financial sources: CNPq, FAPESP, and CAPES

${ }^{1}$ Research performed at Laboratório de Farmacologia Autonômica e Cardiovascular (LabFAC), Department of Pharmacology, Universidade Federal de São Paulo (UNIFESP), Brazil. Part of PhD degree thesis, Postgraduate Program in Pharmacology. Tutor: Prof. Dr. Afonso Caricati-Neto.

This is an Open Access article distributed under the terms of the Creative Commons Attribution License, which permits unrestricted use, (cc) BY distribution, and reproduction in any medium, provided the original work is properly cited. 